\title{
Notiz über eine Farbreaktion der Eiweißkörper. Von
}

S. Edlbacher.

(Ans dem physiologischen Institut Heidelberg.)

(Der Redaktion zugegangen am 4. April 1919.)

Gelegentlich einer Untersuchung, über welche in dieser Zeitschrift noch berichtet werden soll, machte ich folgende Beobachtung: Schüttelt man eine Eiweißlösung mit Natronlauge und Dimethylsulfat und unterschichtet nach der Zersetzung des letzteren das Reaktionsgemisch mit konzentrierter Schwefelsäure, so tritt an der Berührungsfläche beider Flüssigkeiten eine schöne blaurote Farbzone auf, die sich beim Mischen durch die ganze Flüssigkeit verbreitet.

Die Färbung ist der bekannten Glyoxylsäurereaktion äußerst ähnlich und es besteht auch eine Übereinstimmung. mit derselben, indem beide Reaktionen sich gegenseitig bedingen.

So trat die Reaktion ein bei: Caseïn, Serumalbumin, Serumglobulin, Eieralbumin (Merck.), Edestin aus Hanfsamen, Globulin aus Kürbissamen, Globin, Bence-Joneschem Eiweißkörper, Hetero-, Proto- und Deuteroalbumose ${ }^{1}$ ).

Negativ fiel die Reaktion aus bei: Gelatine, Zein, Clupein, Salmin, .Sturin, Esocin, Scombrin; also bei Eiweißkörpern, welche auch die Glyoxylsäureprobe nicht geben.

Aus diesem Grunde scheint das Tryptophan die Reaktion zu bedingen und in der Tat entsteht beim Schütteln einer Tryptophanlösung mit Dimethylsulfat und Natronlauge

1) Es handelte sich um Deuteromyosinose. 
und nachheriger Unterschichtung mit konzentrierter Schwefelsäure dieselbe amethystfarbige Zone.

Indol und Skatol geben nur Rotfärbung, letzteres, wie bekannt, auch ohne Dimethylsulfat.

Ob sich aus den Proteïnen bei der Behandlung mit dem genannten Reagens vielleicht Glyoxylsäure selbst oder ähnliche Körper bilden, kann natürlich noch nicht beantwortet werden.

Bemerkenswert ist noch, daß die Reaktion empfindlicher ist als die Bromreaktion auf freies Tryptôphan. 